\title{
The Herbal Compound Flor-Essence Promotes Step-Down Learning of Mice with High-Energy Diet
}

\author{
Qi Wang ${ }^{1 \#}$, Chao $\mathbf{Q u}^{1 \#}$, Hongyan $\mathrm{Li}^{1}$, Yejun Zhang ${ }^{1}$ and Wei Zou ${ }^{1,2 *}$ \\ ${ }^{1}$ School of Life Science, Liaoning Normal University, China \\ ${ }^{2}$ Liaoning Key Lab of Biotechnology and Molecular Medicine Development, China \\ "These authors contributed equally to this study and share first authroship
}

Submission: November 13, 2018; Published: December 06, 2018

*Corresponding author: Wei Zou, Liaoning Normal University, Xishan Campus, Liaoning Normal University, No. 1 Liushu South Street, Ganjingzi District, Dalian, Liaoning, China

\begin{abstract}
Introduction: Flor-Essence (FE) is an herbal tea with eight ingredients. And it has many functions such as enhancing immunity and antitumor. This article aims to investigate the effect of Flor-Essence on high-energy diet mice.

Methods: The mice were fed with high-fat and high-sugar for 22 days. The blood glucose level and liver index were measured by Blood glucose meter. The exploratory ability and learning and memory ability of the mice were tested by open field and platform experiments.

Results: Short-term high-fat and high-sugar feeding increased blood glucose in mice and significantly damaged liver compared with CTRL; Flor-Essence significantly reduced blood sugar, I inhibited liver damage and reduced liver coefficient. High-energy diets can significantly reduce learning ability, and Flor-Essence can improve learning and memory in high-energy diet mice.
\end{abstract}

Conclusion: Flor-Essence can impede the changes of blood sugar in high-energy diet rats protect against the liver damage and enhance learning ability.

Keywords: High blood glucose; Liver damage; Learning; Memory; Blood sugar; High-energy; Diet; Rats

Abbreviations: HED: High Energy Diet; FE: Flor-Essence; CHOL: Cholesterol; TG: Triglyceride

\section{Introduction}

High-energy diets refer to a class of foods that mainly contain higher levels of fats and/or carbohydrates. Studies have shown that high-energy diet can cause animal fat cells to proliferate excessively, thus producing obesity. Obesity can further cause impaired glucose tolerance, insulin resistance, dyslipidemia and diabetes [1-4]. Long-term consumption of high-fat and high-sugar foods can cause metabolic disorders, hyperglycemia, hyperlipidemia, liver and kidney damage, which causes abnormal metabolism of carbohydrates, proteins and fats, and high triglyceride blood in type 2 diabetes [5]. The pathological changes of hypercholesterolemia are similar. Therefore, the development of nutritional synthetic agents that can replace high-energy diets has attracted more and more attention to assisting medical products.

Flor-Essence (FE) is a food-grade natural herbal formula exclusively produced by Flora, Canada [6]. FE, also known as "Nurse Water", is an herbal tea consisting of eight herbs including Arctium lappa L, Ulmus rubra Muhl, and Sheep sorrel (Rumex acetosella L.), Rheum palmatum L, Cnicus benedictus L, red clover (Trifolium pratense L.), Nasturtium officinale R. Br, kelp
(Laminaria digitata Lmx.). Ammar Saleem et al., [7] demonstrated that bioactive substances in FE are mainly phenolic substances, including phytosterols, terpenoids, saponins and plant isoflavones. Flor-Essence Tonics are commercially available complex mixtures of herbal extracts sold as dietary supplements and often used by cancer patients based on anecdotal evidence of positive benefit $[8,9]$. In a recent survey at a large integrative cancer center in the United States, 38\% of patients reported using herbs and overall, $4.9 \%$ used the Flor-Essence tonic [10]. Flor-Essence tonic is manufactured in Canada where approximately forty thousand units of tonic and dried herbs are distributed to customers in Canada, the United States and internationally each month [11].

Studies have shown that the terpenoids contained in FE mainly have antibacterial, anti-inflammatory, anti-viral, anti-oxidation, antihypertensive, weight loss and other pharmacological effects. FE is used as a nutritional supplement for the prevention of chronic diseases and the improvement of symptoms, but the intervention of FE on blood glucose suppression and learning and memory disorders caused by high-energy diet has not been reported yet. In this paper, Kunming mice were used as research 
objects to establish a high-energy diet model of mice. The changes of various functions of mice in the model group were observed. The effect of FE on high-energy diet mice was discussed. The abnormality and health caused by FE in preventing high-fat and high-sugar were observed. The application in protection provides theoretical and experimental basis.

\section{Materials and Methods}

\section{Animal and supplementation with FE}

Kunming mice, 6-8 weeks old, were used in this experiment, half male and half female, with an average body weight of $27 \mathrm{~g}$, all purchased from SPF Laboratory Animal Center of Dalian Medical University. Mice were randomized into 3 groups: control group $(n=8)$, high energy diet group $(n=8)$, and FE treatment group $(n=8)$.

Twenty-four mice were randomly divided into 3 groups, 8 mice in each group, half male and half female. The control group (C) was given ordinary blocks, purchased from the SPF laboratory animal center of Dalian Medical University, fed with conventional feed, and double distilled water. High-energy diet group (HED), fed with high-fat diet containing 10\% lard, 90\% conventional feed, and water containing $5 \%$ sucrose, and double-distilled water.

The FE treatment group (FE) from Flora company of Canada, was fed daily with the above-mentioned high-fat and high-sugar and was also administered with FE. The FE-treated mice were intragastric ally administered at a dose of $8.5 \mu \mathrm{L}$ of the body weight per gram of body weight. The other groups of mice were given the same volume of double distilled water daily for 22 consecutive days.

\section{Blood sugar assay}

After 16 days, 20 days, and 21 days High energy diet feeding, the mice were fasted for 8-12 hours, massaged the tail vein of the mice, and quickly smashed the tail of the mouse with a blood collection needle, taking blood, and letting the blood flow naturally, and dripping quickly. A blood glucose meter was used to measure the glucose concentration in the peripheral blood of the peripheral vein of each group of mice.

\section{Blood lipid assay}

After 22 days of gavage, the mice were fasted for 8 hours on an empty stomach, and the mice were removed from the eyeballs for blood collection. The obtained blood was allowed to stand at room temperature for $2 \mathrm{~h}$ or $37^{\circ} \mathrm{C}$ for $1 \mathrm{~h}$, and then placed at $4^{\circ} \mathrm{C}$ for $3 \mathrm{~h}$, the blood was coagulated, and the blood clots were shrunk and centrifuged at $4000 \mathrm{rpm}$ for $10 \mathrm{~min}$ to separate the serum. Cholesterol (CHOL) and Triglyceride (TG) were detected using an automated biochemical detector.

\section{Organ index assay}

The body weight (g) of the mice was weighed. The mice were sacrificed by cervical dislocation. Open the abdominal cavity of the mouse with a small pair of scissors in a sterile clean bench. Remove the liver, and the kidneys, and weigh the weight separately. The mean organ index of each group of mice was calculated according to the following formula: organ index = organ weight $(\mathrm{mg}) /$ body weight $(\mathrm{g})$.

\section{Open field test}

The open field test is a method that reflects the behavior and mood of the mouse in the new environment and can be used to test the "excited" or "depressed" state of the central nervous system of the animal [12]. The market experiment box is a $100 \mathrm{~cm} \times 100 \mathrm{~cm} \times 50 \mathrm{~cm}$ box with 25 equal squares on the bottom of the box. The open field experiment was carried out for $5 \mathrm{~min}$ [13]: the mice were placed in the center of the experiment box. The time of the mouse in the central grid and the activity in the open field were observed and recorded.

\section{Step-down test}

This experiment used a hopping recorder. The parameters were set before the experiment, the timing was $5 \mathrm{~min}$, and the voltage was set to $32 \mathrm{~V}$. The mice were placed in separate platforms on each reaction tank. The mice were free to move for $3 \mathrm{~min}$ to familiarize them with the experimental environment and let the mice have a short memory. Cover the cover with a transparent air hole and press the start button. The stainless-steel grid below the reaction tank will immediately pass $32 \mathrm{~V}$. The mice were electrically stimulated, and the mice were stunned by electric shocks, looking for a platform to avoid electric shocks until they found and jumped onto the platform. When the infrared detector signal on the platform changes, the display shows that the latency parameter starts counting. The time when the mouse first jumped off the bottom of the reaction tank from the platform was the incubation period. If the mouse did not jump off the platform within $5 \mathrm{~min}$, the incubation period was calculated as $5 \mathrm{~min}$ and the total number of shocks of the mice after the incubation period was recorded. The number of shocks the mouse received was the number of errors.

\section{Statistical analysis}

The measurement data is expressed as mean \pm SD, and statistical test was performed using SPSS 13.0 software. Intergroup = comparison with variance test. Correlation analysis used multiple linear regression.

\section{Results}

\section{Effect of FE on blood sugar and blood lipids in high energy diet mice}

The blood glucose of the mice was measured at 16 days, 20 days, and 21 days, and there was no significant difference between the groups. At 22 days, the mice were sacrificed to detect cholesterol and triglyceride, and there was no significant difference between the groups.

After 21 days of high-fat and high-sugar feeding, the blood glucose in the high-energy diet group was slightly higher than that of the control group, and the cholesterol and triglyceride values have no difference. The blood glucose level of FE-treated mice was lower than that of the high-energy diet group, but cholesterol 


\section{Current Research in Diabetes \& Obesity Journal}

and triglyceride had no significant difference. The results suggest that high-energy diets in the short-term have an effect on glucose metabolism, and FE can decrease the level of blood glucose in

Table 1: Effect of FE on blood sugar and blood lipids in high energy diet mice.

\begin{tabular}{|c|c|c|c|c|c|}
\hline & \multicolumn{3}{|c|}{ Blood sugar $(\bar{x} \pm$ S $)$} & CHOL & TG \\
\hline & $\mathbf{1}$ & $\mathbf{2}$ & $\mathbf{3}$ & 2.435 & 0.645 \\
\hline Control & $6.7 \pm 1$ & $7.3 \pm 0.2$ & $6.9 \pm 0.2$ & 2.855 & 0.68 \\
\hline HED & $6.5 \pm 0.6$ & $7.4 \pm 1$ & $7.4 \pm 1.3$ & 2.32 & 0.67 \\
\hline FE & $5.8 \pm 0.6$ & $5.1 \pm 0.3$ & $6.7 \pm 0.7$ & & \\
\hline
\end{tabular}

\section{Effect of FE on liver and kidney in mice}

Long-term consumption of high-fat and high-sugar can cause metabolic disorders, hyperglycemia, hyperlipidemia, liver and high-energy diet mice and keep glucose metabolism stable. No effect on lipid metabolism (Table 1). kidney damage, which is associated with abnormal metabolism of carbohydrates, protein and fat, and hypertriglyceridemia in type 2 diabetes. The pathological changes in hypercholesterolemia are

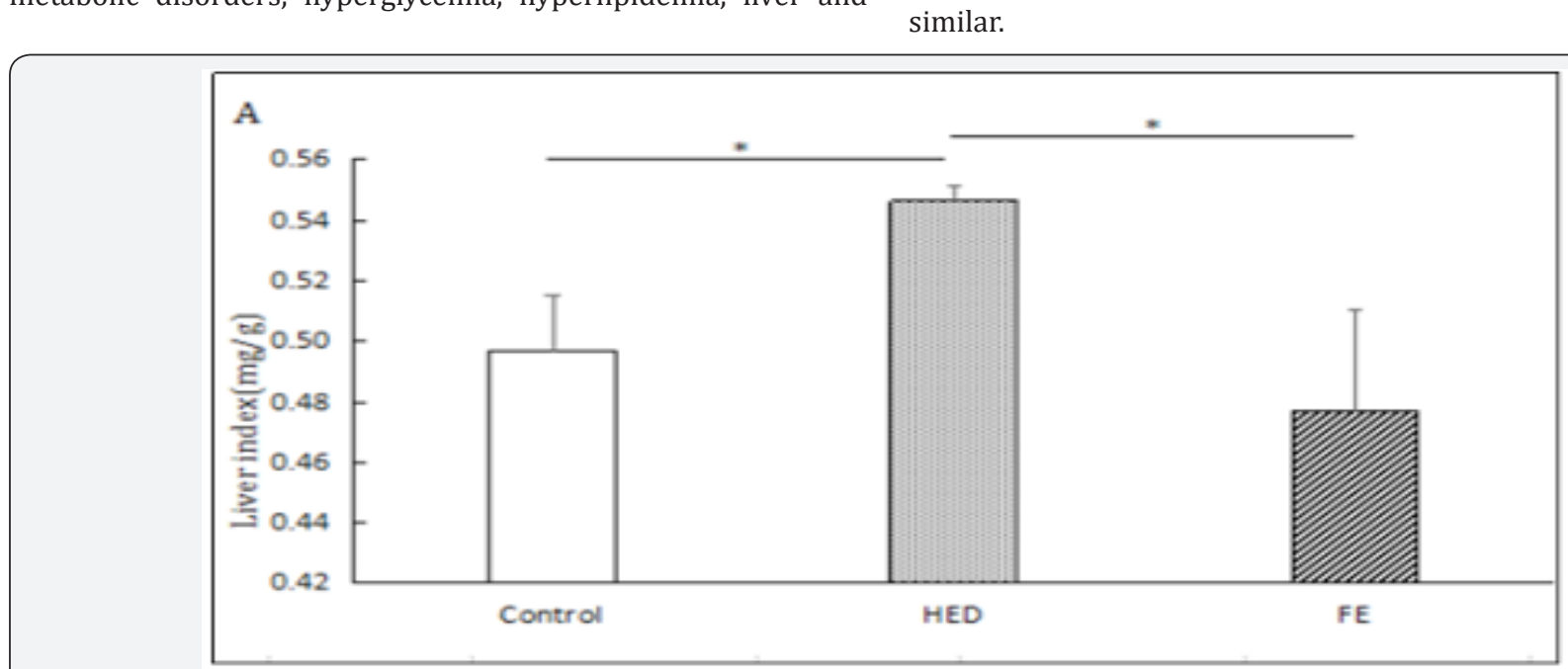

similar.

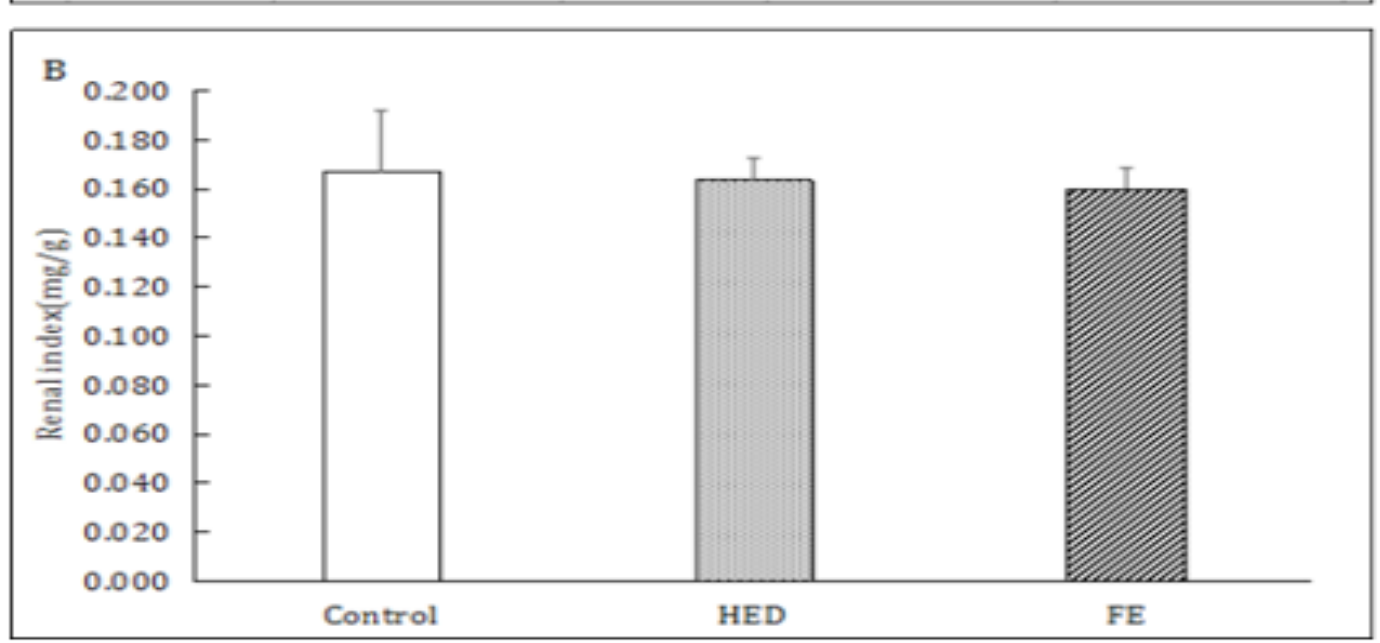

Figure 1A \& 1B: Effect of FE on liver and kidney in mice. After 22 days of feeding, the liver index (A) and renal index (B) of the mice were determined. The liver index of the high-energy diet group (HED) mice was significantly higher than that of the control group. Compared with the simple high-energy diet, the liver index of the FE group (FE) mice with high energy diet was significantly lower. However, there was no significant difference in renal index between the three groups. The results showed that FE significantly affected the liver index during high-energy diet and had no significant effect on the renal index. For all data, n: 3. Data presented as mean and *: represents statistical significance $(p<0.05)$.

Compared with the control group, the liver index of the high-energy diet group was significantly increased (Figure 1A). Compared with the high-fat and high-sugar diet group, the liver index of FE-treated mice was significantly lower (Figure 1A), while the renal index was not significantly different (Figure
1B). It can be shown that the high-fat and high-sugar diet has a significant effect on the liver of mice, but not on the kidney. The results suggest that FE has a significant protective effect on the liver of mice with high-fat and high-sugar diet. 


\section{Current Research in Diabetes \& Obesity Journal}

Effect of FE on spatial exploration ability of high energy diet mice

Obesity, high-calorie diet, lack of physical activity, and aging are the most important factors in inducing type 2 diabetes. Persistence in physical exercise is also one of the ways to treat diabetes. Therefore, this study used open field experiments to analyze whether FE affects the exploration movement of mice. Within 5 minutes, the total distance of movement in the FE group was greater than that in the high-fat, high-sugar-fed mice, and the movement speed was faster (Figure 2). Thus, FE can promote the spontaneous movement of mice fed with high fat and high sugar.
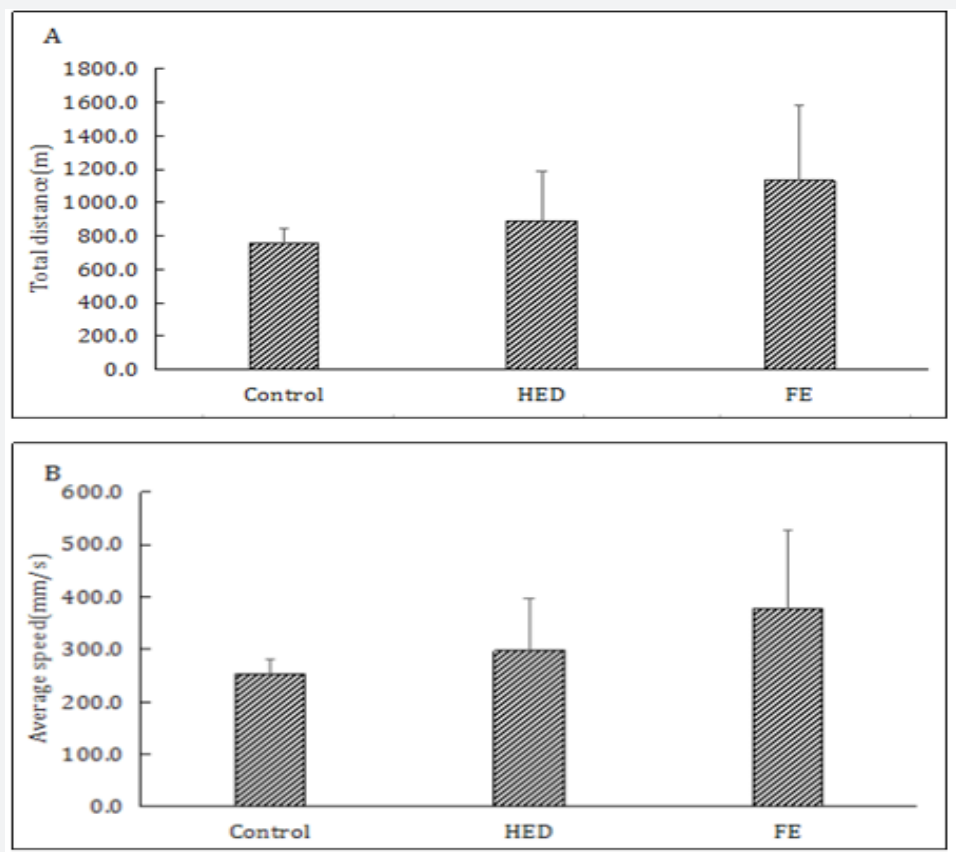

Figure 2A \& 2B: Effect of FE on the total distance and average speed of high fat and high glucose diet mice in open field exercise. The total distance of exercise in the high-energy diet mice was higher than that in the control group. The total distance of FE-fed mice was higher than that in the high-energy diet group, but there was no significant difference(A). High-energy diet mice exercise faster than the control group, and FE-fed mice move faster (B). For all data, n: 3.

\section{Effects of FE on learning and memory in high energy} diet mice.

Table 2: Effect of FE on learning and memory.

\begin{tabular}{|c|c|c|c|c|}
\hline \multirow{2}{*}{ Group } & \multicolumn{2}{|c|}{ Learning Ability (Day 1) } & \multicolumn{2}{c|}{ Memory Ability (Day 2) } \\
\cline { 2 - 5 } & $\begin{array}{c}\text { Latency } \\
\text { Time }\end{array}$ & $\begin{array}{c}\text { Number } \\
\text { of Errors }\end{array}$ & $\begin{array}{c}\text { Latency } \\
\text { Time }\end{array}$ & $\begin{array}{c}\text { Number of } \\
\text { Errors }\end{array}$ \\
\hline Control & $7.67 \pm 1.53$ & $9 \pm 2.65$ & $15 \pm 6.66$ & $11.33 \pm 5.51$ \\
\hline HED & $25.33 \pm 13.84^{*}$ & $6.83 \pm 3.92$ & $18.16 \pm 14.50$ & $8.5 \pm 4.88$ \\
\hline FE & $5.5 \pm 0.71^{*}$ & $3.5 \pm 3.55$ & $15 \pm 11.31$ & $4.5 \pm 0.71$ \\
\hline
\end{tabular}

The latency of the mice in the high-energy diet group was significantly $\left({ }^{*} p<0.05\right)$ higher than that of the control group on the first day of testing; Mice with a high-energy diet and fed FE had significantly $\left({ }^{*} \mathrm{p}<0.05\right)$ lower latency during the first day of testing than the high-energy diet. For all data, $\mathrm{n}: 3$.

Diabetes can lead to disorders of glucose metabolism and vascular disease, damage to the central nervous system, pathological changes, and subsequent development of diabetic encephalopathy. The main clinical manifestations are learning, memory loss and cognitive dysfunction. Compared with the control group, the ability of learning and memory was weak in the high-energy diet group, and the learning ability was significantly decreased $\left({ }^{*} \mathrm{p}<0.05\right)$ (Table 2$)$. The FE group learning and memory ability are better than the high-energy diet group. Improved, the learning effect is significant $\left({ }^{*} p<0.05\right)$ (Table 2$)$. This reminds us that FE can improve the negative effect of high-energy diet on learning and memory.

\section{Discussion}

Type 2 diabetes (T2D) has become a major medical challenge in all countries [14]. In the United States, there are more than 78.6 million obese adults and approximately 29.1 million people suffer from T2D $[15,16]$. T2D is a degenerative disease characterized by insulin resistance and disrupted glucose and lipid metabolism. Insulin resistance prior to the development of T2D is a major cause of other obesity-related metabolic disorders such as T2D and fatty liver disease, dyslipidemia and cardiovascular disease [17].

In recent years, the use of natural plants to prevent and improve diseases has become more and more interesting. FlorEssence (FE), also known as the "nurse tea", is widely used in North American cancer patients and it is an exclusive production of food grade natural herbal formula products made by Flora company of Canada. It contains eight kinds of herbs, which are burdock root (Arctium lappa L.), inner bark of slippery elm Ulmus (rubra Muhl.), sheep sorrel (Rumex acetosella L.), Turkish rhubarb root (Rheum palmatum L.), blessed thistle (Cnicus Benedictus L.), red clover (Trifolium pratense L.), watercress (Nasturtium officinale R.Br) and kelp (Laminaria digitata Lmx.) [7]. It has been 


\section{Current Research in Diabetes \& Obesity Journal}

reported that FE can improve the quality of life and inhibit tumor growth in cancer patients. Experimental studies on a variety of herbs have demonstrated that FE has many biological activities including antioxidant, anti-estrogen, immunostimulatory and anti-tumor effect [18].

Flor-Essence has emerged as a functional food and has many functions such as anti-oxidation and immunity enhancement. Studies have shown that a high-fat diet is associated with hyperlipidemia and coronary heart disease [19]. High-sugar diets are prone to obesity and can also induce insulin resistance, manifested as hyperinsulinemia and elevated blood pressure $[20,21]$. In addition, the metabolism of sugar and lipids interacts with each other [22]. In this study, we simulate a high-energy diet in daily by feeding mice were with high-energy diet life.

The results showed that the blood glucose was higher in the high energy diet group than the normal group. FE could significantly reduce the blood glucose of the high-energy diet group. Thus, FE can improve the increase of blood glucose in high energy diet. What's more, FE have no effect on normal control mice. This result suggests that FE can be used as a daily drink for people with high energy intake.

Metabolic disorders and microvascular disease can cause abnormal brain function and form diabetic encephalopathy. With the increasing number of T2D patients, hypertension, coronary heart disease is also increasing. Furthermore, the influence of obesity and T2D on brain structure and function has been well established and shows a higher risk of cognitive decline and even dementia [23]. Previously, our lab induced T2D in mice by using alloxan monohydrate. We found that diabetes caused Spatial cognitive impairment in mice, which is mediated by different Subtypes of Estrogen Receptor (ER $\alpha$ ) in the Hippocampus. Here, we analyze the impact of high-energy diets on animal learning and memory by step-down method, and anxiety by open field method. The result showed that - high-energy diets was able to decrease learning and memory [24]. In addition, high-energy diets can also induce mice anxiety. Interestingly, FE not only decrease the concentration of blood glucose, it is also improving learning and memory induced by high-energy diets.

The liver is the main part of insulin action and insulin catabolism. The function of liver is closely related to sugar, fat and protein metabolism [25]. High-energy diets can easily cause fatty liver and affect liver function [26,27]. The increase of fat, sugar and protein in the dietary structure of China, especially the increase of animal food, has led to an increase in the incidence of fatty liver [28]. The experimental results show that FE has an inhibitory effect on the increase of liver coefficient in mice, indicating that FE can improve the abnormal symptoms of liver in mice fed with high-energy feed to a certain extent. Therefore, the study can evaluate the effects of high-energy diet on the health of mice from the liver index.

According to reports in the literature, long-term high-fat and high-sugar feeding will develop into obese rats, and there will be brain tissue damage [29]. This experiment used the platform test to test the learning and memory ability of mice and analyze the brain health of high-energy diet mice. The experimental results show that FE has a reduced ability to respond to high-energy diet mice, and the number of errors increases. This result indicates that FE can improve the learning and memory in high-energy diet mice.

\section{Conclusion}

FE has a certain inhibitory effect on the increase of blood glucose in mice and has a health-care function to help lower blood sugar. FE can improve the abnormal symptoms of the liver and the decline of learning and memory ability induced by high energy diet in mice. These results provide a theoretical basis for the wider application of FE health products.

\section{References}

1. English L, Lasschuijt M, Keller KL (2015) Mechanisms of the portion size effect. What is known and where do we go from here? Appetite 88: 39-49.

2. Livingstone MB, Pourshahidi LK (2014) Portion size and obesity. Adv Nutr 5(6): 829-834

3. AJ Tulloch, S Murray, R Vaicekonyte, NM Avena (2015) Neural responses to macronutrients: hedonic and homeostatic mechanisms. Gastroenterology 148(6): 1205-1218.

4. Yi Zhang, Ju Liu, Jianliang Yao, Gang Ji, Long Qian, et al. (2014) Obesity: pathophysiology and intervention. Nutrients 6(11): 5153-5183.

5. Chander AP, Reddy RA, Puchchakayala G (2011) Hypoglycemic and Antidiabetic Activity of Glochidion velutinum on StreptozotocinNicotinamide Induced Type 2 Diabetic Rats. Eur J Biol Sci 3(4): 126130.

6. Carmen Tamayo, MA Richardson, Suzanne Diamond (2000) The Chemistry and Biological Activity of Herbs Used in Flor-Essence Herbal Tonic and Essiac. Phytother Resarch 14(1): 1-14.

7. A Saleem, B Walshe-Roussel, C Harris, M Asim, C Tamayo, et al. (2009) Characterization of Phenolics in Flor-Essence-a compound Herbal product and its Contributing Herbs. Phytochemical Analysis 20(5): 395-401.

8. Swee Keong Yeap, Abdul Rahman Omar, Boon Kee Beh, Noorjahan Banu Alitheen (2013) Rhaphidophora korthalsii modulates peripheral blood natural killer cell proliferation, cytokine secretion and cytotoxicity. Bmc Complementary \& Alternative Medicine 13(1): 145-145.

9. Kulp KS, Montgomery JL, Nelson DO, Cutter B, Latham ER, et al. (2006) Essiac and Flor-Essence herbal tonics stimulate the in vitro growth of human breast cancer cells. Breast Cancer Research \& Treatment 98(3): 249-259.

10. Richardson MA, Ramirez T, Palmer JL, Greisinger A, Singletary SE (2018) Complementary/alternative medicine use in a comprehensive cancer center and the implications for oncology. J Clin Oncol 18(13): 2505-2514.

11. Seely D, Kennedy DA, Myers SP, Cheras PA, Lin D, et al. (2007) In Vitro Analysis of the Herbal Compound Essiac. Anticancer Res 27(69): 38753882.

12. A Kato-Kataoka, M Sakai, R Ebina, C Nonaka, T Asano, et al. (2010) Soybean-derived phosphatidylserine improves memory function of the elderly Japanese subjects with memory complaints. J Clin Biochem Nutr 47(3): 246-255

13. Shen J, Qu C, Xu L, Sun H, Zhang J (2018) Resveratrol exerts a protective effect in chronic unpredictable mild stress-induced depressivelike behavior: involvement of the AKT/GSK3 $\beta$ signaling pathway in hippocampus. Psychopharmacology. 


\section{Current Research in Diabetes \& Obesity Journal}

14. Chen L, Magliano DJ, Zimmet PZ (2012) The worldwide epidemiology of type 2 diabetes mellitus-present and future perspectives. Nat Rev Endocrinol 8(4): 228-236.

15. Centers for Disease Control and Prevention (2017) National Diabetes Statistics Report: estimates of diabetes and its burden in the United States, 2017. US Department of Health and Human Services, Atlanta.

16. Ogden CL, Lamb MM, Carroll MD, Flegal KM (2010) Obesity and socioeconomic status in adults: United States, 2005-2008. NCHS Data Brief (50): 1-8.

17. Seidell JC (2000) Obesity, insulin resistance and diabetes-a worldwide epidemic. Br J Nutr 83(S1): 5-8.

18. Cheung S, Lim KK, Tai J (2005) Antioxidant and anti-inflammatory properties of ESSIAC and Flor-Essence. Oncol Rep 14(5): 1345-1350.

19. Oliver MF (1987) Dietary fat and coronary heart disease. Br Heart 58(5): 423-428.

20. Reaven GM (1991) Insulin resistance and compensatory hyperinsulinemia: Role in hypertension dyslipidemia and coronary heart disease. Am Heart J 121(4 Pt 2): 1283-1288.

21. El Hafidi M, Valdez R, Banos G (2000) Possible relationship between altered fatty acid composition of serum, platelets and aorta and hypertension induced by sugar feeding in rats. Clin Exp Hypertens 22(1): 99-108.

22. Budohoski L, Panczenko-Kresowska B, Langfort J, Zernicka E Dubaniewicz A, et al. (1993) Effects of saturated and polyunsaturated fat enriched diet on the skeletal muscle insulin sensitivity in young rats. Physiol Pharmacol 44(4): 391-398.

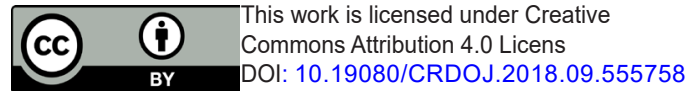

23. S Kullmann, M Heni, M Hallschmid, A Fritsche, H Preissl, et al (2016) Brain Insulin Resistance at the Crossroads of Metabolic and Cognitive Disorders in Humans. Physiol Rev 96(4): 1169-209.

24. MR Zhang, C Qu, J Sun, C Wang, HY Li, et al (2017) Different subtypes of estrogen receptor $\alpha$ and related signal molecules in the hippocampus are associated with spatial cognitive impairment of diabetic mice. Sheng li xue bao 69(3): 252-260.

25. Gábor, Firneisz (2014) Non-alcoholic fatty liver disease and type 2 diabetes mellitus: The liver disease of our age? World Journal of Gastroenterology 20(27): 9072-9089.

26. J Guo, H Tao, A Alasadi, Q Huang, S Jin (2017) Niclosamide piperazine prevents high-fat diet-induced obesity and diabetic symptoms in mice[J]. Eating and Weight Disorders - Studies on Anorexia, Bulimia and Obesity (Suppl 1): 1-6.

27. G Fouret, S Gaillet, I Lecomte, Beatrice Bonafos, F Djohan, et al (2018) 20-Week follow-up of hepatic steatosis installation and liver mitochondrial structure and activity and their interrelation in rats fed a high-fat-high-fructose diet. British Journal of Nutrition 119(4): 368380 .

28. Jesse G Meyer, Samir Softic, Nathan Basisty, Matthew J Rardin, Eric Verdin, et al. (2018) Multi-Omic Profiling Reveals the Opposing Forces of Excess Dietary Sugar and Fat on Liver Mitochondria Protein Acetylation and Succinylation. bio Rxiv.

29. Jun Gao, Lorraine Ghibaudi, Margaret Van Heek, Joyce J Hwa (2002) Characterization of diet-induced obese rats that develop persistent obesity after 6 months of high-fat followed by 1 month of low-fat diet. Brain Research 936(1): 87-90.

Your next submission with Juniper Publishers
will reach you the below assets
- Quality Editorial service
- Swift Peer Review
- Reprints availability
- E-prints Service
- Manuscript Podcast for convenient understanding
- Global attainment for your research
- Manuscript accessibility in different formats
( Pdf, E-pub, Full Text, Audio)
- Unceasing customer service
Track the below URL for one-step submission
https://juniperpublishers.com/online-submission.php

